\title{
Immunogenicity of Oxford-AstraZeneca COVID-19 Vaccine in Vietnamese Health-Care Workers
}

\author{
Nguyen Van Vinh Chau, ${ }^{1 *}$ Lam Anh Nguyet, ${ }^{2}$ Nguyen Thanh Truong, ${ }^{1}$ Le Mau Toan, ${ }^{1}$ Nguyen Thanh Dung, ${ }^{1}$ Le Manh Hung, ${ }^{1}$ \\ Mai Thanh Nhan, ${ }^{1}$ Dinh Nguyen Huy Man, ${ }^{1}$ Nghiem My Ngoc, ${ }^{1}$ Huynh Phuong Thao, ${ }^{1}$ Tran Nguyen Hoang Tu, ${ }^{1}$ \\ Huynh Kim Mai, ${ }^{3}$ Do Thai Hung, ${ }^{3}$ Nguyen Thi Han Ny, ${ }^{2}$ Le Kim Thanh, ${ }^{2}$ Nguyen To Anh, ${ }^{2}$ Nguyen Thi Thu Hong, ${ }^{2}$ \\ Le Nguyen Truc Nhu, ${ }^{2}$ Lam Minh Yen, ${ }^{2}$ Marc Choisy, ${ }^{2,4}$ Tran Tan Thanh, ${ }^{2}$ Guy Thwaites, ${ }^{2,3}$ and Le Van Tan ${ }^{2 *}$ \\ for the Oxford University Clinical Research Unit COVID-19 Research Group \\ ${ }^{1}$ Hospital for Tropical Diseases, Ho Chi Minh City, Vietnam; ${ }^{2}$ Oxford University Clinical Research Unit, Ho Chi Minh City, Vietnam; ${ }^{3}$ Institute of \\ Pasteur, Nha Trang City, Vietnam; ${ }^{4}$ Centre for Tropical Medicine and Global Health, Nuffield Department of Medicine, University of Oxford,
} Oxford, United Kingdom

\begin{abstract}
We studied the immunogenicity of the Oxford-AstraZeneca vaccine in health-care workers of a major infectious diseases hospital in Vietnam. We measured neutralizing antibodies before and 14 days after each dose, and at day 28 and month 3 after dose 1. A total of 554 workers (136 men and 418 women; age range, 22-71 years; median age, 36 years) participated with the study. Of the 144 participants selected for follow-up after dose 1, 104 and 94 gave blood for antibody measurement at weeks 6 and 8, and at month 3 after dose 1, respectively. The window time between the two doses was 6 weeks. At baseline, none had detectable neutralizing antibodies. After dose 1, the proportion of participants with detectable neutralizing antibodies increased from $27.3 \%$ (151 of 554) at day 14 to $78.0 \%$ (432 of 554) at day 28 . Age correlated negatively with the development and the levels of neutralizing antibodies. However, at day 28 , these differences were less profound, and women had a greater seroconversion rate and greater levels of neutralizing antibodies than men. After dose 2, these age and gender associations were not observable. In addition, the proportion of study participants with detectable neutralizing antibodies increased from $70.2 \%$ (73 of 104) before dose 2 (week 6, after dose 1) to $98.1 \%$ (102 of 104) 14 days later. At month 3, neutralizing antibodies decreased and $94.7 \%$ (89 of 94) of the study participants remained seropositive. The Oxford-AstraZeneca COVID-19 vaccine is immunogenic in Vietnamese health-care workers. These data are critical to informing the deployment of the COVID-19 vaccine in Vietnam and in Southeast Asia, where vaccination coverage remains inadequate.
\end{abstract}

\section{INTRODUCTION}

Severe acute respiratory syndrome coronavirus 2 (SARSCoV-2) is the cause of the ongoing COVID-19 pandemic. ${ }^{1}$ Since its first detection in Wuhan, China, in late 2019, SARSCoV-2 has now become an endemic virus globally. The vaccine is thus the most plausible approach to return to prepandemic life. As such, vaccine development has ramped up globally during the past year. As of June 1, 2021, 185 and 102 vaccine candidates are under the pre-clinical and clinical development phases, respectively. ${ }^{2}$ In addition, seven vaccines have received WHO approval for emergency use. ${ }^{2}$

Approved vaccines have been rapidly deployed globally. As of July 26, 2021, more than 3.8 billion doses of COVID-19 vaccines have been administered worldwide. Vietnam received the first doses of the Oxford-AstraZeneca vaccine in early March 2021. As of July 18, 2021, more than 4 million doses have been administered in Vietnam, the majority of which included the Oxford-AstraZeneca vaccines. ${ }^{3}$

Although a vaccine must fulfill the required efficacy criteria to receive an approval for use in humans, the rapid development and deployment of COVID-19 vaccines worldwide necessitate follow-up studies to understand more fully the development and persistence of vaccine-induced immunity in different populations. Such knowledge is critical to inform global vaccination strategies and the development of nextgeneration vaccines.

Despite the current surge, which has been escalating since the second week of May 2021, Vietnamese people remained relatively naive to SARS-CoV-2 infections. ${ }^{4,5}$ As of

*Address correspondence to Nguyen Van Vinh Chau or Le Van Tan, 764 Vo Van Kiet, District 5, Ho Chi Minh City, Vietnam. E-mails: chaunvv@oucru.org and tanlv@oucru.org
July 18, 2021, 31,391 polymerase chain reaction-confirmed cases have been reported in Vietnam - a country of more than 97 million people. ${ }^{3}$ Therefore, Vietnam is an ideal setting for a vaccine evaluation study because the results naturally reflect the immunity induced by COVID-19 vaccines. There has been no report about the immunogenicity of the OxfordAstraZeneca COVID-19 vaccine from Southeast Asia. We studied the immunogenicity of the Oxford-AstraZeneca COVID-19 vaccine in a cohort of 554 health-care workers in an infectious diseases hospital in southern Vietnam.

\section{METHODS}

Setting and COVID-19 vaccine rollout in Vietnam. Our study was conducted at the Hospital for Tropical Diseases (HTD) in Ho Chi Minh City. HTD is a 550-bed tertiary referral hospital for patients with infectious diseases (including COVID-19) in southern Vietnam. ${ }^{6}$ Between December 2020 and February 2021, $\approx 40$ COVID-19 patients were treated at HTD.

Vietnam received the first 117,000 doses of the OxfordAstraZeneca COVID-19 vaccine in early March 2021. The time window between the two doses was set for a minimum of 4 weeks, with some variation (4-12 weeks), depending on the availability of the vaccine. According to the Vietnamese Ministry of Health, high-risk groups, especially frontline health-care workers, were prioritized for vaccination (Supplemental Materials). HTD staff members were eligible for vaccination and were the first in Vietnam to receive a COVID-19 vaccine in March 2021.

Data collection. We collected demographic information and $3 \mathrm{~mL}$ of blood from the study participants. Blood sampling was scheduled for a total of eight time points, including one before and seven after dose 1 . The seven time points 
TABLE 1

Demographics of the study participants

\begin{tabular}{|c|c|c|c|c|c|c|c|}
\hline \multirow[b]{2}{*}{ Demographic } & \multirow[b]{2}{*}{ Whole group $(N=554)$} & \multirow[b]{2}{*}{ Subgroup $1(n=144)$} & \multirow[b]{2}{*}{ Subgroup $2(n=104)$} & \multirow[b]{2}{*}{ Subgroup $3(n=94)$} & \multicolumn{3}{|c|}{ Comparison between groups } \\
\hline & & & & & $\mathrm{P}^{*}$ & $\mathrm{P} 2 \dagger$ & P3ł \\
\hline Male, $n(\%)$ & $136(25)$ & $31(22)$ & $25(24)$ & $21(22)$ & 0.449 & 0.912 & 0.644 \\
\hline Female, $n(\%)$ & $418(75)$ & $113(78)$ & $79(76)$ & $73(78)$ & & & \\
\hline Median age, y (range)† & $36(22-71)$ & $37(24-65)$ & $37(24-5)$ & $37(24-65)$ & 0.136 & 0.213 & 0.154 \\
\hline \multicolumn{8}{|l|}{ Age group, y } \\
\hline $20-39, n(\%)$ & $332(60)$ & $79(55)$ & $57(55)$ & $50(53)$ & 0.313 & 0.621 & 0.471 \\
\hline $40-60, n(\%)$ & 217 (39) & $64(44)$ & $46(44)$ & $43(46)$ & & & \\
\hline $61-71, n(\%)$ & $5(1)$ & $1(1)$ & $1(1)$ & $1(1)$ & & & \\
\hline
\end{tabular}

$\mathrm{Px}=P$ values per subgroup

* Comparison between the whole group and subgroup 1.

† Comparison between whole group and subgroup 2 .

$\ddagger$ Comparison between whole group and subgroup 3 .

after dose 1 included weeks 2, 4, 6, and 8; and months 3, 6 and 12. Week 6 and 8 after dose 1 corresponded to the time points of before and 14 days after dose 2 , respectively.

After week 4 of the first dose, because of resource constraints, blood sampling was narrowed down to a subgroup of 144 randomly selected individuals, matching for age and gender with the whole-group study participants (Table 1). Our report focuses on the period from baseline to month 3 after the first dose.

Neutralizing antibody measurement. Neutralizing antibodies were measured using a U.S. Food and Drug Administration Emergency Use Authorization-approved assay: namely, the SARS-CoV-2 surrogate virus neutralization test (GenScript, Singapore). Prior to testing, plasma samples were first diluted $1: 10$ and then inactivated at $56^{\circ} \mathrm{C}$ for 30 minutes. The experiments were carried out according to the manufacturer's instructions. Results are expressed as a percentage of inhibition, using a cutoff of $30 \%$. This cutoff was applied successfully in the original report. ${ }^{7}$ The percentage of inhibition measured by the SARS-CoV-2 surrogate virus neutralization test has been shown to correlate well with the neutralizing antibody titers measured by the conventional plaque reduction neutralization assay. ${ }^{7}$

Neutralizing antibody data from cases of natural infection. To compare the development of neutralizing antibodies induced by vaccination against that of natural infection, we included data from 11 Vietnamese patients who had mild or asymptomatic infections. Details about these individuals and neutralizing antibody measurements are detailed in our recent report. $^{8}$

Statistical analysis. We used Fisher's exact, the $\chi^{2}$, or the Mann-Whitney $U$ test to compare between groups (when appropriate). Logistic regression was used to assess the association between age and the probability of having detectable neutralizing antibodies. Linear regression was used to assess the association between age and neutralizing antibodies levels. The analyses were carried using Prism 9.0.2 (graphpad.com, San Diego, CA).

Ethical approval. The study was approved by the Institutional Review Board of HTD and the Oxford Tropical Research Ethics Committee, University of Oxford, UK. Written informed consent was obtained from all participants.

\section{RESULTS}

Demographics of the study participants. A total of 649 of 894 HTD staff members $(72.6 \%)$ consented to participate in the vaccine evaluation study. Five hundred fifty-four of 649 participants $(85.4 \%)$ were monitored successfully up to day 28 after the first dose and were thus included for analysis as a whole group. The 554 study participants were between 22 and 71 years of age (median age, 36 years). Women were predominant, accounting for $75.4 \%$ of study participants (418 of 554) (Table 1).

Of the 144 participants in the subgroup, 104 (72.2\%) and $94(65.3 \%)$ were monitored successfully up to 14 days after the second dose (i.e., week 8 after dose 1) and 3 months (week 13) after the first dose, respectively. The age and gender distributions of these subgroups are comparable with that of the whole group (Table 1). The time window between the first and the second dose was 6 weeks.

Development of detectable neutralizing antibodies. At baseline, none of the 104 study participants in the subgroup had detectable neutralizing antibodies (Table 2). At days 14 and 28 after the first dose, the proportion of study participants with detectable neutralizing antibodies increased from

TABLE 2

The proportion of study participants with detectable neutralizing antibodies after vaccination

\begin{tabular}{|c|c|c|c|c|c|c|c|c|}
\hline \multirow[b]{2}{*}{ Time point } & \multicolumn{4}{|c|}{ Whole group } & \multicolumn{4}{|c|}{ Subgroup } \\
\hline & Total $(N=554)$ & Male $(n=136)$ & Female $(n=418)$ & $P$ value ${ }^{*}$ & Total $(N=104)$ & Male $(n=25)$ & Female $(n=79)$ & $P$ value ${ }^{*}$ \\
\hline Baseline, $n(\%)$ & 0 & 0 & 0 & NA & 0 & 0 & 0 & NA \\
\hline 14 Days after dose $1, n(\%)$ & $151(27.3)$ & $40(29.4)$ & $111(26.6)$ & 0.52 & $31(29.8)$ & $10(40.0)$ & $21(26.6)$ & 0.20 \\
\hline 28 Days after dose $1, n(\%)$ & $432(78.0)$ & $97(71.3)$ & $335(80.1)$ & 0.031 & $82(78.8)$ & $20(80.0)$ & $62(78.5)$ & 0.87 \\
\hline Before dose 2, $n(\%)$ & NA & NA & NA & NA & 73 (70.2) & $17(68.0)$ & $56(70.1)$ & 0.78 \\
\hline 14 Days after dose $2, n(\%)$ & NA & NA & NA & NA & $102(98.1)$ & $24(96.0)$ & $78(98.7)$ & 0.43 \\
\hline Month 3 after the first dose $\dagger$ & NA & NA & NA & NA & $89(94.7)$ & $21(95.5)$ & 68 (94.4) & 1 \\
\hline
\end{tabular}

$\mathrm{NA}=$ not applicable.

* For comparison between men and women.

$\dagger N=94$ (22 men and 72 women) 
A

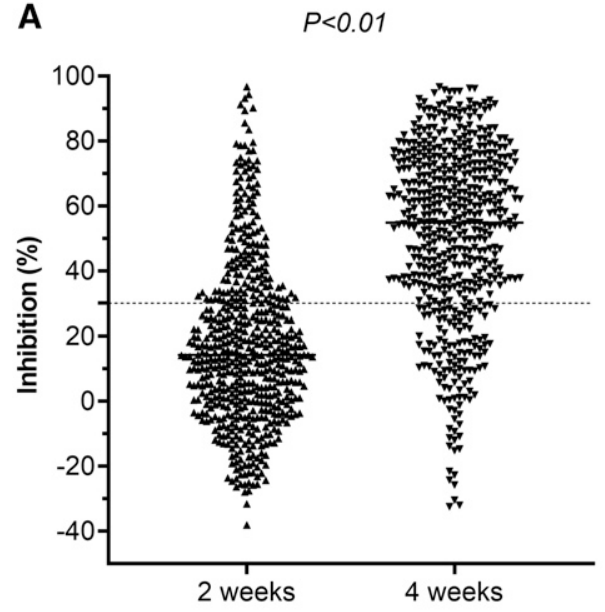

B

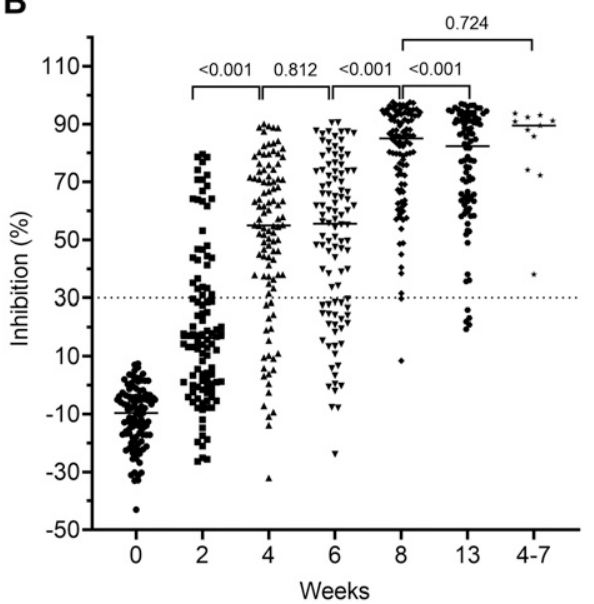

FIGURE 1. Development of neutralizing antibodies levels after vaccination. (A) Neutralizing antibody levels measured at 2 and 4 weeks after the first dose was administered to 554 study participants. (B) Neutralizing antibody levels measured at time points from baseline to month 3 after the first dose was administered to the subgroups. Data on neutralizing antibody levels obtained from 11 convalescent sera collected during weeks 4 through 7 (right column) from cases with mild or asymptomatic infection were included as references.

$27.3 \%$ (151 of 554 ) to $78.0 \%$ (432 of 554 ), respectively, among all 554 individuals of the whole group. The proportion of study participants with detectable neutralizing antibodies reached $98.1 \%$ (102 of 104) 14 days after the second dose, and then decreased slightly to $94.7 \%$ (89 of 94) at month 3 after the first dose (Table 2).

Kinetics of neutralizing antibody levels. After the first dose, neutralizing antibody levels measured at day 28 were significantly greater than those measured on day 14 (Figure 1A), but comparable with those measured at week 6 (Figure 1B). On day 14 after the second dose, neutralizing antibodies increased significantly and were comparable with those levels obtained from Vietnamese people with asymptomatic or mild infection (Figure 1B). At month 3 after the first dose, neutralizing antibody levels were significantly less than those measured at 14 days after the second dose (Figure 1B).

Neutralizing antibodies versus age and gender. On day 14 after the first dose, the development and levels of detectable neutralizing antibodies among the 554 study participants correlated negatively with age. This difference was less profound at day 28 after dose 1, especially with regard to the development of detectable neutralizing antibodies (Figure 2). At these corresponding time points, similar trends were also observed among individuals of the subgroup, but the difference was not significant (Figure 3 and Supplemental Figure 1), likely because of the small sample size. At 14 days after the second dose (week 8 after dose 1) and month 3 after the first dose, the proportion of individuals with detectable neutralizing antibodies was similar across age groups (Supplementa1 Figure $1 \mathrm{~B}$ and $\mathrm{C})$.

In terms of gender, with the exception of day 28 after the first dose, neutralizing antibody levels and the proportion of study participants with detectable neutralizing antibodies were comparable between men and women (Table 2 and Supplemental Figure 2).
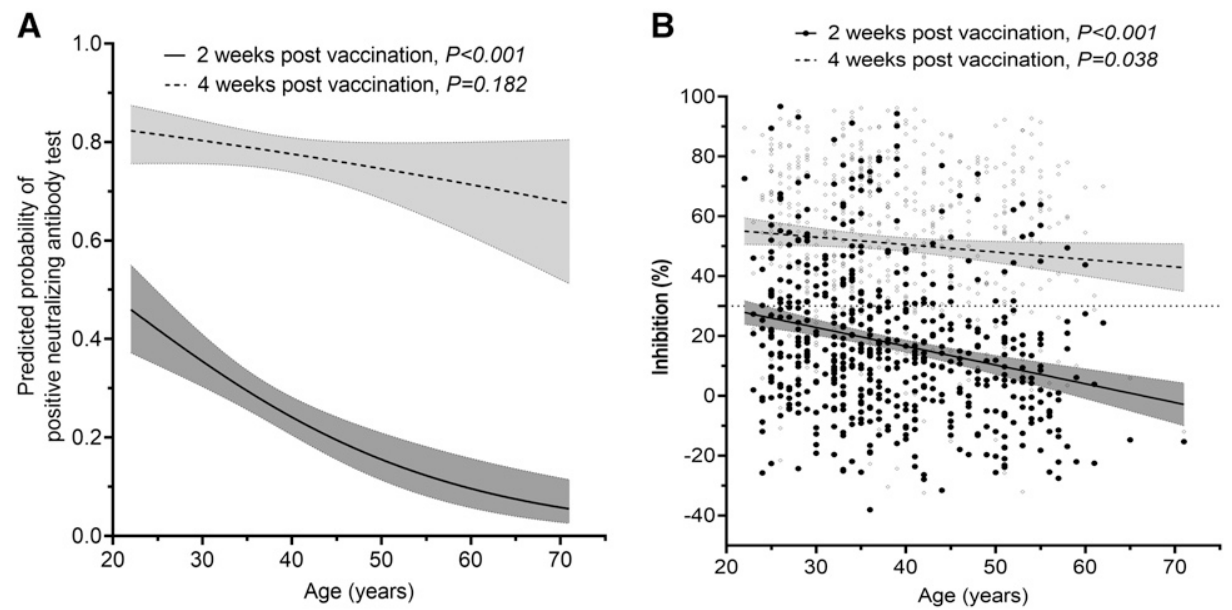

FIGURE 2. The associations between neutralizing antibody level and age. (A) Association between age and the probabilities of having detectable neutralizing antibodies at 2 and 4 weeks after the first does was administered to 554 study participants. (B) Association between age and neutralizing antibody levels measured at 2 and 4 weeks after the first dose was administered to 554 study participants. Black circles represent data for the 14-day time point; gray circles represent data for 28 -day time point. Shaded areas indicate $95 \%$ Cls. 
A
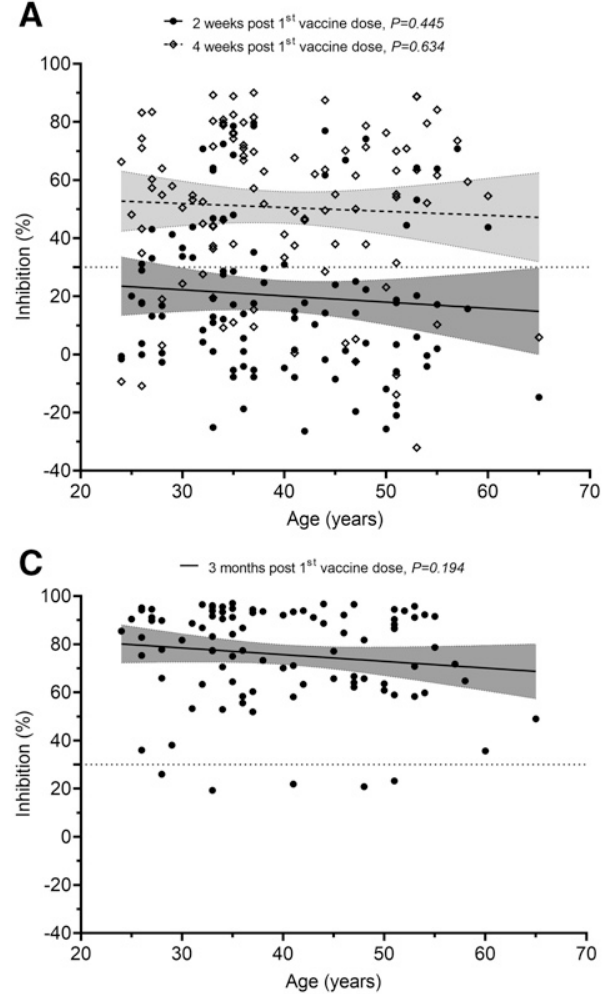

B

-6 weets post $1^{12}$ vaccine dose, $P=0.336$

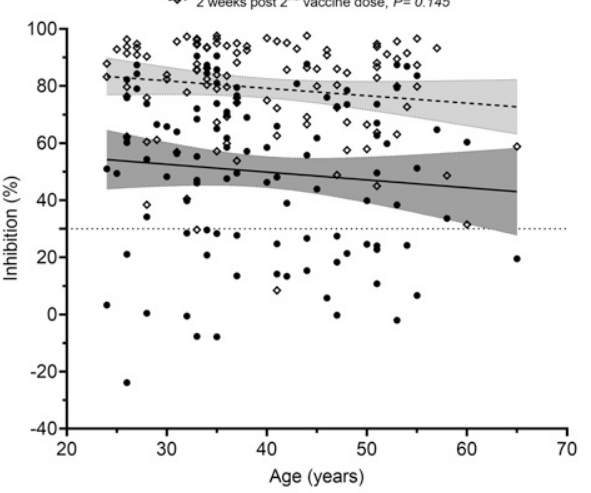

Figure 3. Neutralizing antibody levels of participants selected to assess the impact of the second dose. (A) Two and 4 weeks after the first dose $(n=104)$. (B) Before the second dose ( 6 weeks after the first dose) and 2 weeks after the second dose $(n=104)$. (C) At month 3 after the first dose $(n=94)$. Shaded areas indicate $95 \%$ Cls.

\section{DISCUSSION}

We report the immunogenicity of the Oxford-AstraZeneca COVID-19 vaccine in a cohort of 554 Vietnamese healthcare workers in a tertiary referral hospital for patients with infectious diseases in southern Vietnam. We show that the Oxford-AstraZeneca COVID-19 vaccine is immunogenic in Vietnamese people. Neutralizing antibodies increased after each dose, with the seroconversion rate reaching $98.1 \%$ (102 of 104) at 14 days after dose 2. At month 3 after dose 1 , neutralizing antibody levels decreased, and $94.7 \%$ (89 of 94) of the study participants remained seropositive.

Findings from the original phase $2 / 3$ trial showed that spike protein-specific IgG developed within 2 weeks after vaccination; and at 14 days after the second dose, its titers increased, with a seroconversion rate of 208 of 209 (> 99\%). ${ }^{9}$ Consistently, our study shows the development and levels of neutralizing antibodies increase significantly after each dose, with the former reaching $98.1 \%$ at 14 days after the second dose. Parallel with these reports are realword data from the United Kingdom showing that the administration of the second dose increased protection against SARS-CoV-2 infection from $65 \%$ by dose 1 to $70 \%$ by dose 2 among vaccine recipients. ${ }^{10}$ A single dose of OxfordAstraZeneca or Pfizer COVID-19 vaccines reduced COVID-19 hospital admissions among vaccine recipients by $88 \%$ and $91 \%$, respectively in Scotland. ${ }^{11}$

Older individuals, especially those 80 years or older, without prior infection had lower levels of neutralizing antibodies induced by the first dose than younger adults. ${ }^{12,13}$ These age-dependent responses were most profound within the first 3 weeks after vaccination, but were resolved by the administration of the second dose. ${ }^{12}$ Although similar trends were observed in our study, at day 28 after the first dose, the differences in our study were negligible, especially in terms of the seroconversion rate. None of our study participants was older than 71 years, which explains why the observed differences were less profound compared with the UK population-based study. ${ }^{10}$

Our results provide reassuring evidence for the effectiveness of the proposed vaccination strategy that aims at prioritizing the first dose for as many people as possible in the first instance. ${ }^{14}$ However, the data also emphasize the importance of the second dose, ${ }^{15}$ especially in older people, and it remains unknown whether the third dose is needed to provide long-term protection. A decline in antibody titers was recorded at weeks 8 through 12 after the first two doses among 75 study participants in the United Kingdom, ${ }^{16}$ but the administration of a third dose helped boost the immune response. Antibody waning is presumably more profound among individuals without prior infection. A follow-up study is therefore critical to assess the levels of antibody waning among our study participants and the correlated level of protection, especially in the context of the rapid spread of the delta variant globally.

A strength of our study is that it was conducted in a naive population, with no prior SARS-CoV-2 infections. ${ }^{4}$ Thus, our data reflect more naturally the immunity profiles induced by the Oxford-AstraZeneca COVID-19 vaccine. In addition, although the correlates of protection for the COVID-19 
vaccine remain to be determined, neutralizing antibodies are considered to be the most reliable surrogates. ${ }^{17}$ Therefore, by measuring neutralizing antibodies, our findings reflect more accurately the potential of correlates of protection.

Our study has some limitations. First, we did not study cellular immunity, especially $\mathrm{T}$-cell response. Cellular immunity has been recognized increasingly to play a role in the pathogenicity and immune response of the SARS-CoV-2 infection. ${ }^{18}$ Therefore, the durability of both cellular and humoral immune responses should be explored further. Second, because of the age and gender structure of the HTD staff, we did not include participants older than 71 years, and women were predominant among our study subjects. Of note, compared with men, women seemed to respond better to the Oxford-AstraZeneca COVID-19 vaccine at day 28 after the first dose, which merits further research.

In summary, the Oxford-AstraZeneca COVID-19 vaccine is immunogenic in Vietnamese health-care workers. Neutralizing antibody levels decreased at month 3 after vaccination. The requirement for a third dose warrants further research. These data are critical to informing the deployment of the COVID-19 vaccine in Vietnam and beyond.

Received August 2, 2021. Accepted for publication November 29, 2021.

Published online January 7, 2022.

Note: Supplemental figures appear at www.ajtmh.org.

Acknowledgments: We thank our colleagues at the Hospital for Tropical Diseases in Ho Chi Minh City, Vietnam, for their participations in this study. OUCRU Vietnam COVID-19 research group: Chambers Mary, Choisy Marc, Day Jeremy, Dong Huu Khanh Trinh, Dong Thi Hoai Tam, Du Hong Duc, Dung Vu Tien Viet, Fisher Jaom, Flower Barney, Geskus Ronald, Hang Vu Thi Kim, Ho Quang Chanh, Ho Thi Bich Hai, Ho Van Hien, Hung Vu Bao, Huong Dang Thao, Huynh le Anh Huy, Huynh Ngan Ha, Huynh Trung Trieu, Huynh Xuan Yen, Kestelyn Evelyne, Kesteman Thomas, Lam Anh Nguyet, Lawson Katrina, Leigh Jones, Le Kim Thanh, Le Dinh Van Khoa, Le Thanh Hoang Nhat, Le Van Tan, Lewycka Sonia Odette, Lam Minh Yen, Le Nguyen Truc Nhu, Le Thi Hoang Lan, Nam Vinh Nguyen, Ngo Thi Hoa, Nguyen Bao Tran, Nguyen Duc Manh, Nguyen Hoang Yen, Nguyen Le Thao My, Nguyen Minh Nguyet, Nguyen To Anh, Nguyen Thanh $\mathrm{Ha}$, Nguyen Than Ha Quyen, Nguyen Thanh Ngoc, Nguyen Thanh Thuy Nhien, Nguyen Thi Han Ny, Nguyen Thi Hong Thuong, Nguyen Thi Hong Yen, Nguyen Thi Huyen Trang, Nguyen Thi Kim Ngoc, Nguyen Thi Kim Tuyen, Nguyen Thi Ngoc Diep, Nguyen Thi Phuong Dung, Nguyen Thi Tam, Nguyen Thi Thu Hong, Nguyen Thu Trang, Nguyen Thuy Thuong Thuong, Nguyen Xuan Truong, Nhung Doan Phuong, Ninh Thi Thanh Van, Ong Phuc Thinh, Pham Ngoc Thanh, Phan Nguyen Quoc Khanh, Phung Ho Thi Kim, Phung Khanh Lam, Phung Le Kim Yen, Phung Tran Huy Nhat, Rahman Motiur, Thuong Nguyen Thi Huyen, Thwaites Guy, Thwaites Louise, Tran Bang Huyen, Tran Dong Thai Han, Tran Kim Van Anh, Tran Minh Hien, Tran Phuong Thao, Tran Tan Thanh, Tran Thi Bich Ngoc, Tran Thi Hang, Tran Tinh Hien, Trinh Son Tung, van Doorn H. Rogier, Van Nuil Jennifer, Vidaillac Celine Pascale, Vu Thi Ngoc Bich, Vu Thi Ty Hang, Yacoub Sophie. HTD COVID-19 research group: Nguyen Van Vinh Chau, Nguyen Thanh Dung, Le Manh Hung, Huynh Thi Loan, Nguyen Thanh Truong, Nguyen Thanh Phong, Dinh Nguyen Huy Man, Nguyen Van Hao, Duong Bich Thuy, Nghiem My Ngoc, Nguyen Phu Huong Lan, Pham Thi Ngoc Thoa, Tran Nguyen Phuong Thao, Tran Thi Lan Phuong, Le Thi Tam Uyen, Tran Thi Thanh Tam, Bui Thi Ton That, Huynh Kim Nhung, Ngo Tan Tai, Tran Nguyen Hoang Tu, Vo Trong Vuong, Dinh Thi Bich Ty, Le Thi Dung, Thai Lam Uyen, Nguyen Thi My Tien, Ho Thi Thu Thao, Nguyen Ngoc Thao, Huynh Ngoc Thien Vuong, Huynh Trung Trieu, Pham Ngoc Phuong Thao, Phan Minh Phuong. EOCRU COVID-19 research group: Bachtiar, Andy, Baird, Kevin J., Dewi, Fitri, Dien, Ragil, Djaafara, Bimandra A.,
Elyazar, Iqbal E., Hamers, Raph L., Handayani, Winahyu, Kurniawan, Livia Nathania, Limato, Ralalicia, Natasha, Cindy, Nuraeni, Nunung, Puspatriani, Khairunisa, Rahadjani, Mutia, Rimainar, Atika, Saraswati, Shankar, Anuraj H., Suhendra, Henry, Sutrisni, Ida Ayu, Suwarti, Tarino, Nicolas, Timoria, Diana, Wulandari, Fitri. OUCRU-NP COVID-19 research group: Basnyat Buddha, Duwal Manish, Gautum Amit, Karkey Abhilasha, Kharel Niharika, Pandey Aakriti, Rijal Samita, Shrestha Suchita, Thapa Pratibha, Udas Summita. The American Society of Tropical Medicine and Hygiene has waived the Open Access fee for this article due to the ongoing COVID-19 pandemic and has assisted with publication expenses.

Financial support: This study was funded by the Wellcome Trust of Great Britain (106680/B/14/Z and 204904/Z/16/Z) and the National Institutes of Health/National Institute of Allergy and Infectious Diseases (HHSN272201400007C, subcontract no. S000596-JHU).

Authors' addresses: Nguyen Van Vinh Chau, Nguyen Thanh Truong, Le Mau Toan, Nguyen Thanh Dung, Le Manh Hung, Mai Thanh Nhan, Dinh Nguyen Huy Man, Nghiem My Ngoc, Huynh Phuong Thao, and Tran Nguyen Hoang Tu, Hospital for Tropical Diseases, Ho Chi Minh City, Vietnam, E-mails: chaunvv@oucru.org, bsthanh truong@gmail.com, drmautoan@gmail.com, bsdungbvbnd@gmail. com, bslemanhhung@gmail.com, mainhanyds@gmail.com, bshuym an@gmail.com, ngocnm@oucru.org, hpt251984@yahoo.com.vn, and bs.hoangtu.bvbnd@gmail.com. Lam Anh Nguyet, Nguyen Thi Han Ny, Le Kim Thanh, Nguyen To Anh, Nguyen Thi Thu Hong, Le Nguyen Truc Nhu, Lam Minh Yen, Tran Tan Thanh, and Le Van Tan, Oxford University Clinical Research Unit, Ho Chi Minh City, Vietnam, E-mails: nguyetla@oucru.org, nynth@oucru.org, thanhlk@oucru.org, anhnt@oucru.org, hongntt@oucru.org, nhulnt@oucru.org, yenlm@ oucru.org, thanhtt@oucru.org, and tanlv@oucru.org. Huynh Kim Mai and Do Thai Hung, Institute of Pasteur, Nha Trang City, Vietnam, E-mails: mai064@yahoo.com and hungdt02@yahoo.com. Marc Choisy, Oxford University Clinical Research Unit, Ho Chi Minh City, Vietnam, and Centre for Tropical Medicine and Global Health, Nuffield Department of Medicine, University of Oxford, Oxford, UK, E-mail: mchoisy@oucru.org. Guy Thwaites, Oxford University Clinical Research Unit, Ho Chi Minh City, Vietnam, and Institute of Pasteur, Nha Trang City, Vietnam, E-mail: gthwaites@oucru.org.

This is an open-access article distributed under the terms of the Creative Commons Attribution (CC-BY) License, which permits unrestricted use, distribution, and reproduction in any medium, provided the original author and source are credited.

\section{REFERENCES}

1. Zhu $\mathrm{N}$ et al., 2020. A novel coronavirus from patients with pneumonia in China, 2019. N Engl J Med 382: 727-733.

2. WHO, 2021. COVID-19 Vaccine Tracker and Landscape. Available at: who.int. Accessed June 7, 2021.

3. Vietnamese Ministry of Health, 2021. An Official Website of Vietnamese Ministry of Health. Available at: ncov.moh.gov.vn. Accessed July 18, 2021.

4. Chau NVV et al., 2021. Absence of SARS-CoV-2 antibodies in health care workers of a tertiary referral hospital for COVID-19 in southern Vietnam. $J$ Infect 82: e36-e37.

5. Van Tan L, 2021. COVID-19 control in Vietnam. Nat Immunol 22: 261.

6. Chau NVV et al., 2020. The natural history and transmission potential of asymptomatic SARS-CoV-2 infection. Clin Infect Dis 71: 2679-2687.

7. Tan CW et al., 2020. A SARS-CoV-2 surrogate virus neutralization test based on antibody-mediated blockage of ACE2-spike protein-protein interaction. Nat Biotechnol 38: 1073-1078.

8. Mai HK et al., 2021. Long-term humoral immune response in persons with asymptomatic or mild SARS-CoV-2 infection, Vietnam. Emerg Infect Dis 27: 663-666.

9. Folegatti PM et al., 2020. Safety and immunogenicity of the ChAdOx1 nCoV-19 vaccine against SARS-CoV-2: a 
preliminary report of a phase $1 / 2$, single-blind, randomised controlled trial. Lancet 396: 467-478.

10. Pritchard E et al., 2021. Impact of Vaccination on SARS-CoV-2 Cases in the Community: A Population-Based Study Using the UK's COVID-19 Infection Survey. MedRxiv. Available at: https://doi.org/10.1101/2021.04.22.21255913.

11. Vasileiou $E$ et al., 2021. Interim findings from first-dose mass COVID-19 vaccination roll-out and COVID-19 hospital admissions in Scotland: a national prospective cohort study. Lancet 397: 1646-1657.

12. Wei $\mathrm{J}$ et al., 2021. The Impact of SARS-CoV-2 Vaccines on Antibody Responses in the General Population in the United Kingdom. MedRxiv. Available at: https://doi.org/10.1101/ 2021.04.22.21255911.

13. Abu Jabal $\mathrm{K}$ et al., 2021. Impact of age, ethnicity, sex and prior infection status on immunogenicity following a single dose of the BNT162b2 mRNA COVID-19 vaccine: real-world evidence from healthcare workers, Israel, December 2020 to January 2021. Euro Surveill 26: 1-5.

14. Romero-Brufau $S$ et al., 2021. Public health impact of delaying second dose of BNT162b2 or mRNA-1273 COVID-19 vaccine: simulation agent based modeling study. BMJ 373: n1087.

15. Lopez Bernal $\mathrm{J}$ et al., 2021. Effectiveness of COVID-19 vaccines against the B.1.617.2 (delta) variant. $N$ Engl J Med 385: 585-594.

16. Flaxman, A., et al., 2021. Tolerability and immunogenicity after a late second dose or a third dose of ChAdOx1 nCoV-19 (AZD1222). Lancet. Available at: http://dx.doi.org/10.2139/ssm.3873839.

17. Khoury DS et al., 2021. Neutralizing antibody levels are highly predictive of immune protection from symptomatic SARSCoV-2 infection. Nat Med 27: 1205-1211.

18. Ogbe A et al., 2021. T cell assays differentiate clinical and subclinical SARS-CoV-2 infections from cross-reactive antiviral responses. Nat Commun 12: 1-14. 